\title{
Traduire
}

Revue française de la traduction

$230 \mid 2014$

À la croisée du texte et de l'image

\section{Traduire au cinéma (Rétrospective)}

\section{Imma Kunz}

Traducteur : Maurice Voituriez

\section{OpenEdition}

\section{Journals}

Édition électronique

URL : http://journals.openedition.org/traduire/622

DOI : $10.4000 /$ traduire. 622

ISSN : 2272-9992

Éditeur

Société française des traducteurs

Édition imprimée

Date de publication : 15 juin 2014

Pagination : 42-62

ISSN : 0395-773X

Référence électronique

Imma Kunz, «Traduire au cinéma (Rétrospective) », Traduire [En ligne], 230 | 2014, mis en ligne le 15 juin 2016, consulté le 19 avril 2019. URL : http://journals.openedition.org/traduire/622 ; DOI : 10.4000/ traduire.622 


\section{Traduire au cinéma (Rétrospective)}

\section{Imma Kunz}

Cette étude, traduite de l'allemand par Maurice Voituriez, a été publiée dans le numéro 139 de Traduire (1989)(1).

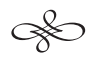

N'ayant pas la télévision et me rendant rarement au cinéma, j'ai franchi allègrement le "seuil d'incompétence " en traduisant partiellement l'étude d'Imma Kunz sur l'activité cinématographique de P.-F. Caillé. J'ai quand même vu les deux grands films qu'elle analyse du point de vue de la synchronisation. Je souhaite au lecteur de trouver autant de plaisir et d'intérêt à connaître ce texte que j'en ai eu à le traduire, passionnant "devoir de vacances".

Maurice Voituriez

\section{Qu'est-ce que la synchronisation d'un film ?}

"... doubler, c'est enregistrer un dialogue traduit en le substituant au "dialogue original" ".

C'est ainsi que P.-F. Caillé définissait l'art de la synchronisation, art qui fit son apparition en même temps que le cinéma parlant.

Si telle est la définition, on peut se demander dans quelle mesure la synchronisation se distingue de la traduction. Tout comme Christian Delmas, Pierre-François Caillé(2) affirme que synchroniser, c'est en effet traduire. Edmond Cary déclare à ce sujet : " le texte écrit ne capte jamais qu'une image incomplète et déformée de la langue. II ne donne qu'une approximation grossière de ce qu'essaie de communiquer l'être humain qui entreprend de s'exprimer à l'aide de ce moyen complexe qu'est le langage " et place ainsi le doublage au-dessus de la traduction proprement dite :

(1) Maurice Voituriez l'a également reprise dans son ouvrage Les Félicités de la Traduction, Chez l'auteur, Paris, 1998.

(2) Une présentation/bibliographie succincte des auteurs cités est donnée en fin d'article.

(Les notes en italiques ont été ajoutées par la rédaction en 2014). 
"Tous les autres genres [de la traduction] ne connaissent qu'une des facettes du langage : le doublage accepte d'être fidèle à toutes. "

\section{Le pour et le contre}

Si on admet le point de vue réaliste de Caillé, une des raisons pour lesquelles les producteurs de films préfèrent souvent la synchronisation au sous-titrage, c'est " ... qu'ils savent très bien qu'un film rapporte plus d'argent en version doublée qu'en version sous-titrée ".

Caillé lui-même avait pratiqué les deux genres et préférait la synchronisation car le public ne possède en général que médiocrement la langue originale et se fatigue rapidement de lire les sous-titres. En revanche Hans Vöge attribue à la synchronisation sa faible connaissance de la langue étrangère car elle n'offre jamais au public l'occasion d'entendre cette dernière.

Vöge signale aussi un aspect non négligeable :

quand on importe beaucoup de films en langue étrangère, le nombre d'acteurs et d'actrices disponibles risque d'être bien inférieur à celui des rôles à doubler. Il en résulte qu'on entend trop souvent les mêmes voix, surtout si, par économie, un acteur double plusieurs rôles dans le même film!

Ce danger existe même dans un grand pays comme la France où on double beaucoup et où on dispose d'une bonne réserve d'acteurs. Je m'en suis aperçue en entendant la remarque d'une Française, certainement dépourvue d'idées préconçues, qui me dit avoir reconnu, dans un film synchronisé la voix d'un acteur doublant un rôle de la série télévisée Texas.

\section{Difficultés et limites de la synchronisation}

\section{Différences syntaxiques}

C'est surtout sur le plan de la syntaxe que les langues allemande et anglaise constituent pour le synchronisateur un véritable casse-tête. Le rejet du verbe à la fin de la proposition subordonnée, l'inversion de l'adjectif par rapport au substantif (royal palace au lieu de "palais royal "), pour ne citer que deux exemples, l'obligent à trouver d'autres solutions, ou bien à renoncer tout bonnement au synchronisme phonétique. II est plus facile de synchroniser mutuellement les langues latines car elles sont analogues dans leur construction et leur étymologie. Pourtant les terminaisons différentes constituent des difficultés particulières. Roma n'est nullement synchrone avec " Rome". 


\section{La langue parlée courante}

"Plus on se rapproche, dit Caillé, de la vie naturelle, de la simplicité, plus la transposition d'une langue dans une autre est compliquée... Nous touchons là à toute la complexité de notre art : rester fidèle tout en demeurant nature ! ».

La langue parlée courante est un des problèmes majeurs du synchronisateur. Les dialogues rapides, accompagnés et soulignés par des gestes appropriés, lui donnent davantage de difficultés que de longs discours cohérents.

\section{La couleur locale}

Dans presque tous les films se pose la question de savoir s'il faut conserver la couleur locale et l'exprimer par des accents et des tournures particulières.

Contrairement à la page imprimée, l'écran nous rappelle constamment de manière visuelle (par les enseignes, les vêtements, les panneaux indicateurs, etc.) que les scènes et les personnages appartiennent à un autre pays. [...] On offre peu de latitude à l'acteur pour réaliser le "type étranger ", en dehors d'un accent exagéré. Les phrases maladroites, les voyelles traînées et autres distorsions voulues seront conçues en vue d'une bonne synchronisation(3).

Dans les cas, peu fréquents, où le script est tiré d'une œuvre écrite dans la langue-cible, le problème se résout de lui-même.

Il en va de même des accents régionaux qui souvent sont perdus à la synchronisation. Thomas L. Rowe écrit à ce sujet :

Même si tout le film est réalisé " avec l'accent ", une oreille non familiarisée ne reconnaîtra pas les variantes régionales et, de toute manière, les connotations locales en matière d'humour, d'exotisme ou d'ethnie échapperont en grande partie au public étranger.

Le synchronisateur joue aussi, lorsqu'il traduit un dialogue de film, le rôle ingrat d'auxiliaire de la censure. Rowe le déplore :

Dans un certain domaine, le rédacteur américain du texte doublé est contraint d'ignorer les intentions de l'auteur. C'est quand il doit assumer la pénible fonction de censeur face à des propos rabelaisiens tels qu'en comportent les scripts français et italiens. Dans quelle mesure il doit s'ériger en défenseur de la morale anglo-saxonne et trahir l'esprit du texte original, cela dépend de l'appréciation du "doubleur " ou du producteur sur les limites du puritanisme du public, ou quelquefois de la tolérance du censeur.

Dans les exemples de films cités plus loin il n'y a pas de censure de ce genre. II aurait été intéressant de savoir ce qu'il en était d'autres films synchronisés par P.-F. Caillé, notamment sur

(3) Thomas L. Rowe. 
des sujets politiques (Le jugement de Nuremberg, parlant français, 1962). Je pense néanmoins qu'en matière de censure - telle que la définit Rowe - la France est bien plus libérale que les pays anglo-saxons(4).

\section{Les limites de la synchronisation}

Un synchronisateur compétent est généralement capable de trouver pour son travail de version les synonymes les mieux adaptés phonétiquement. Pourtant il n'existe quelquefois aucun synonyme et le seul mot correct ne s'adapte pas phonétiquement au texte original. Dans pareils cas le traducteur le plus habile sera contraint de faire des concessions, dans un sens ou dans un autre.

On se heurte également aux limites de la synchronisation quand il s'agit d'humour. Quelle décision doit prendre le traducteur s'il est possible de traduire mais si la traduction n'est pas phonétiquement synchrone? Rowe pense qu'alors :

L'unique ressource du traducteur est de créer une phrase synchrone aussi amusante que l'original. C'est en matière de comédie que le travail du doubleur s'écarte le plus du travail du traducteur.

Qu'il s'agisse de pure traduction ou de synchronisation de passages ironiques ou humoristiques, le charme de l'original est malheureusement perdu pour l'essentiel. "Comme l'argot, l'humour est prisonnier de son propre langage „(5).

Dans le même esprit, Caillé déclare :

Le traducteur a toujours la ressource d'ajouter en désespoir de cause une note en bas de page (" jeu de mots intraduisible en français "), tandis que le dialoguiste, lui, est bien forcé de trouver une solution.

Malheureusement le traducteur de dialogues de films dispose généralement de peu de temps pour trouver la solution idéale.

En effet la synchronisation d'un film n'est pas du ressort de la production mais de la distribution et " les facteurs commerciaux et la hâte jouent un rôle capital dans la distribution d'un film ".

Même dans des conditions idéales, c'est-à-dire avec suffisamment de temps et d'argent, István Fodor estime qu'un doublage parfait n'est pas imaginable.

Avant d'aborder la technique de P.-F. Caillé et les critères d'une critique de la synchronisation, j'aimerais citer encore P.-F. Caillé :

(4) Je ne partage pas ce point de vue qui, à mon avis, n'est exact qu'en matière de morale, non en matière idéologique ou historique $-\mathrm{N}$. du T.

(5) István Fodor. 
Le cinéma est en fin de compte une fabrique d'illusions. Disons que le doublage cherche à donner l'illusion d'une illusion. Et n'allons pas trop loin sur ce terrain dont la qualité ne doit pas être si mauvaise après tout puisque des auteurs de renom daignent à présent s'y risquer.

\section{La technique de la traduction}

On a souvent décrit en détail la technique selon laquelle Caillé et ses confrères français traduisaient les dialogues de films originaux. Je voudrais voir ici de plus près la technique de traduction utilisée en synchronisation et selon laquelle Caillé travaillait également. Pour la synchronisation d'un film de cinéma (10 bobines, environ 1 heure 30) il faut de 2 à 10 jours, montage compris.

La durée dépend essentiellement de l'abondance des dialogues. On peut traiter plus rapidement des documentaires ou des films où on fait appel à un récitant (ou à un commentateur) car ce dernier n'apparaît généralement pas à l'écran et on n'a pas à se préoccuper du mouvement de ses lèvres.

Comment s'attaque-t-on à cette tâche ? Après avoir vu le film, on commence à écrire au crayon sur la " bande rythmo(6) " la traduction. Le nouveau texte - qu'il se trouve au-dessus ou audessous du texte original - doit se trouver exactement à l'endroit où il doit être parlé ultérieurement car déjà un écart d'un demi centimètre rendrait le travail dénué de toute valeur, le texte n'étant plus synchrone.

Les vraies difficultés ne font alors que commencer. Le traducteur doit faire face simultanément à plusieurs exigences, souvent quasi contradictoires. On lui demande en effet " de dire ce qu'on dit dans l'original et en même temps de mettre des " $M$ " sous des " $M$ " et des " OU " sous des "OU ", de passer du dialogue humoristique au sentimental, de faire parler des gangsters et des duchesses "(7). Le synchronisateur doit toujours tenir compte du fait que dans différentes langues les mêmes voyelles se prononcent différemment. Rowe exprime cela de façon imagée : " II faut, d'une manière ou d'une autre, faire passer le chameau sémantique par le chas de l'aiguille phonétique ".

Que faire si un texte présente de telles difficultés ? "Eh bien, on triche... " répondait Caillé quand on lui posait la question. II donnait comme exemple la scène de Autant en emporte le vent où Scarlett, entourée d'admirateurs, à qui on demande qui aura la faveur de lui apporter

(6) Jean-François Cornu, qui publie prochainement aux PUR Le doublage et le sous-titrage. Histoire et esthétique (voir l'entretien en page 10) nous signale ici une imprécision. Le traducteur travaille en effet sur la "bande-mère", laquelle sert ensuite à la confection de la bande rythmo proprement dite.

(7) Quand aucun nom d'auteur n'est mentionné, les citations sont de P.-F. Caillé. 
un morceau de gâteau, répond, après un temps de réflexion : I... I... I think Charles Tarlinton. En prononçant le "th ", elle montrait le bout de la langue. Alors que P.-F. Caillé mentionne seulement ce problème, son ami et confrère Cary cite également la solution : " Fort judicieusement, il l'avait résolu par un jeu de scène, par une "grimace" et non par un mot ".

En dehors de la possibilité de ne pas traduire un mot, on peut aussi introduire des mots qui n'existaient pas. Normalement le processus de la parole commence par l'ouverture de la bouche et un petit mouvement des lèvres pour que la bouche soit prête à former le premier son. À la fin du processus de la parole la bouche revient en position de repos par un autre mouvement muet.

Très souvent on peut donc glisser du texte sur des mouvements de bouche où il n'y a, en fait, rien de proféré dans la version originale.

Si les particularités du film le permettent, il existe encore une autre possibilité de "tourner " les difficultés phonétiques:

On fait parler hors champ un personnage qui ne dit rien parce qu'on ne pouvait pas traduire ce qui se disait en gros plan(8).

La vitesse d'élocution, différente d'une langue à l'autre, donne un moyen de surmonter des problèmes de traduction. Non seulement le texte français est plus long que l'anglais, mais encore il est dit plus vite. Si on transpose la vitesse d'élocution de l'anglais en français, on obtient un accent traînant du genre "Western ".

Pour y parer on en est fréquemment réduit à adopter délibérément un compte de syllabes différent, faire coïncider deux syllabes avec trois ou inversement, de manière à accrocher une articulation française à une articulation initialement toute différente(9).

Ce sont là quelques " astuces " simples et toujours utilisables en synchronisation. II en existe d'autres qui servent moins à résoudre des problèmes qu'à pratiquer une sorte de censure. Un ami et confrère de Caillé, dont je préfère ne pas donner le nom, me parla d'un film où, pour des raisons purement diplomatiques, les " mauvais " Japonais devenaient en version synchronisée de " mauvais " Chinois, tandis que les " bons " Chinois devenaient de "bons Japonais". Pour des yeux européens la différence n'est pas toujours évidente...

II n'est pas rare, non plus, que plusieurs traducteurs travaillent sur un seul et même film. C'est ainsi que les films Vingt mille lieues sous les mers et Le jugement de Nuremberg furent synchronisés en partie par Caillé, en partie par Charles Goldblatt. Ce dernier m'expliqua en détail leur manière de travailler.

\footnotetext{
(8) Delmas.

(9) Cary.
} 
Il est bon de savoir qu'un synchronisateur n'a pas nécessairement besoin de maîtriser la languesource. À la différence des traducteurs littéraires, il peut procéder à la transcription phonétique en partant d'une traduction fidèle(10).

Quels que soient les efforts du traducteur et aussi réussi que soit son texte, à tous égards, bien souvent un film ne sera pas parfaitement synchronisé et il n'en sera pas responsable. En effet, si une des nombreuses étapes de la synchronisation est bâclée, toutes les autres perdent de leur valeur et le résultat n'est pas satisfaisant.

\section{Critères pour une critique}

P.-F. Caillé - lors du 6e Congrès de la FIT à Montréal - répondait en ces termes à la question de savoir ce que signifiait en synchronisation le concept de qualité :

Il y a deux éléments à considérer : la qualité technique et la qualité pure. C'est là que nous rejoignons les traducteurs. II faut être synchrone, certes, mais il faut aussi être fidèle à la pensée de l'auteur.

Fodor qui considère la synchronisation, non d'un point de vue pratique mais avec les yeux d'un analyste, distingue les critères de :

- synchronisme textuel (inhaltliche Synchronität),

- synchronisme interne (charakterliche Synchronität)(11),

- synchronisme phonétique (phonetische Synchronität).

En pratique on ne peut pas toujours considérer ces trois critères séparément car ils s'influencent mutuellement.

\section{Synchronismes textuel et interne}

Le synchronisme textuel demande d'abord la fidélité au texte-source mais le synchronisateur doit aussi tenir compte d'autres caractéristiques du dialogue original. Généralement des textes de même teneur ont une longueur différente d'une langue à l'autre. Nous retombons donc sur le critère du synchronisme phonétique.

Si le texte-cible est plus court que le texte-source, on peut résoudre le problème en faisant du "remplissage ". Seulement, de l'anglais au français, c'est le contraire qui se produit, ce qui peut

(10) J.-F. Cornu précise que si cette pratique existait effectivement à l'époque de P.-F. Caillé (sans être systématique), elle a été abandonnée depuis au moins trente ans.

Il est toutefois intéressant de signaler que cette pratique est parfois utilisée au théâtre, au moins en GrandeBretagne, comme le déplorait William Gregory dans Traduire, $n^{\circ} 222$, http://traduire.revues.org/433

(11) Traduction de Cary. 
donner une mutilation du texte et une dénaturation de sa teneur. Non seulement, les textes français sont plus longs que les anglais mais encore - nous l'avons déjà indiqué - ils sont dits plus vite. On peut en tirer profit pour résoudre certaines difficultés de traduction.

L'œil humain normal peut percevoir et distinguer correctement les mouvements de la bouche correspondant à neuf sons oraux par seconde. II s'ensuit que si on parle plus vite, la possibilité de "lire " la parole tombe pratiquement à zéro(12).

Le synchronisme textuel est donc réalisable par le biais de la manipulation phonétique mais on court le risque de négliger le synchronisme interne :

La vitesse est un facteur personnel. Une élocution lente ou rapide peut renseigner sur la personnalité de celui qui parle. Si le ralentissement ou l'accélération de la parole, produits artificiellement, contredisent les gestes et le maintien, étroitement associés à l'élocution, alors il peut en résulter un pénible déphasage(13).

Par synchronisme interne, Fodor entend surtout le timbre personnel de la voix, l'intonation, la vitesse et le niveau sonore de la parole, qui sont en rapport étroit avec les gestes et le maintien.

Selon lui, les acteurs chargés de la synchronisation doivent, si possible, " rejouer " les scènes des films, cela pour assurer un meilleur synchronisme interne. Edmond Cary considérait également le synchronisme interne comme aussi important que le synchronisme phonétique :

Un coup de poing soulignant le mot clé peut flanquer par terre une traduction dont toutes les labiales collent à merveille, s'il tombe à contresens dans la phrase traduite.

Cary montre ainsi comment l'acteur est absolument " tenu " par son texte et Caillé est bien d'accord avec lui : l'acteur, le comédien, ne peut donner sa mesure et avant tout " jouer juste " que si le texte qu'il a à interpréter est lui-même naturel.

Si le texte est naturel et " accroche " le public, alors le synchronisme phonétique ne joue, selon Caillé, qu'un rôle secondaire :

Pourvu qu'une phrase soit au rythme, qu'elle traduise l'original avec toute sa charge de sensibilité, de colère ou de tendresse, qu'elle en garde la saveur, il n'est pas nécessaire, sauf dans certains cas de très gros plans, que toutes les labiales soient en place.

S'il faut faire des concessions à l'un ou l'autre critère, Caillé donne sans hésiter la priorité aux synchronismes textuel et interne. Tout comme Fodor, il estime que la qualité du texte compense le déphasage phonétique. En revanche, Delmas attribue au synchronisme phonétique une place bien plus importante, sans mettre en cause pour autant la qualité de la traduction : « le respect des aspects extérieurs doit être considéré comme plus important que la littéralité de la traduction ".

(12) Fodor.

(13) Fodor. 
Cette position peut s'expliquer par le fait que Delmas travaille au Canada où l'influence des États-Unis est forte. Alors que les Français préfèrent un synchronisme optiquement parfait et constituent, avec les Italiens, le public le plus tolérant en matière de films synchronisés, les Américains - ainsi que les Anglais et, dans une moindre mesure, les Allemands - sont les découvreurs les plus critiques des déphasages phonétiques.

\section{Synchronisme phonétique}

Les synchronismes textuel et interne sont des notions largement subjectives et ne permettent pas une critique objective de la synchronisation.

Le synchronisme phonétique, par contre, peut être analysé grâce à des systèmes de notation spécialement conçus à cette fin. Fodor a élaboré un schéma sur l'aptitude du spectateur à "lire " un texte par le mouvement des lèvres de l'acteur, cela pour des positions différentes de la caméra. Ces positions seront indiquées par la suite par leurs abréviations anglaises :

- $\mathrm{CU}=$ close-up

- MS = medium shot

- LS = long shot

- $\mathrm{FA}=$ front angle

- $\mathrm{SA}=$ side angle

- $\mathrm{BA}=$ back angle

Aperçu de la perceptibilité des sons pour différentes positions de caméra CU/FA

\section{Consonnes :}

On peut discerner les labiales (bilabiales et labiodentales) de toutes les autres consonnes. Si on articule bien, on peut aussi distinguer les bilabiales $(p, b, m)$ des labiodentales $(f, v)$.

Voyelles :

Toute voyelle ouverte est discernable. Dans les voyelles médianes et fermées on peut distinguer les labiales des non labiales. Par exemple on peut distinguer [ i ] de [ u ] et [ y ], [ e ] et $[E]$ de $[0]$ et $[\varnothing](14)$ et distinguer toutes celles-là des autres voyelles et consonnes. On peut aussi reconnaître la différence entre voyelles et consonnes. On peut aussi reconnaître la différence entre les voyelles ouvertes ([ i ], [ u ], [ y ]) et fermées [ a ], [ a ], etc.). Ces voyelles aussi peuvent être distinguées des autres voyelles et consonnes.

(14) Ce sont les symboles de l'Association de phonétique internationale. 
Les sons médians sont perçus comme ouverts ou fermés. Plus une voyelle est ouverte, mieux elle est perçue. Les voyelles fermées ([ i ], [ U ]) sont difficilement distinguables de ([ j ], $[\mathrm{g}])$.

\section{CU/SA}

On peut reconnaître les voyelles ouvertes des autres voyelles et de toutes les consonnes.

\section{CU/BA}

On ne discerne que le début et la fin du " discours".

\section{MS/FA}

Ici la discernabilité dépend principalement de l'ouverture de la bouche lors de l'articulation.

Les consonnes labiales peuvent être discernées de toutes les autres consonnes et voyelles. On distingue les voyelles ouvertes des fermées. Les voyelles médianes sont vraisemblablement perçues comme ouvertes ou fermées. On discerne bien en tous cas le début et la fin du "discours".

\section{$\mathrm{MS} / \mathrm{BA}$}

On ne discerne que le début et la fin du " discours".

\section{LS/FA}

On reconnaît les consonnes et les voyelles, ainsi que le début et la fin du "discours ".

\section{$\mathrm{LS} / \mathrm{SA}$}

On ne discerne que le début et la fin du " discours".

\section{$\mathrm{LS} / \mathrm{BA}$}

On ne discerne que le début et la fin du "discours ".

$[\ldots]$

\section{L'effet sur le public}

Comment réalise-t-on un film synchronisé ?

L'analyse théorique du synchronisme phonétique permet de découvrir les défauts. Comme je l'ai déjà mentionné, on peut jouer de la vitesse d'élocution ou faire appel à différentes astuces.

Selon moi le but de la synchronisation est de présenter au public un film aussi impeccable que possible sur les plans interne et phonétique et de donner l'impression qu'il a été tourné dans la langue du public. Telle est aussi l'opinion de Delmas : 
Un texte synchrone doit être le plus discret possible : à partir du moment où l'auditeur dit : "Tiens, c'est bien trouvé ce mot-là ! ", c'est perdu, ça décroche. Les grandes trouvailles sont celles qui passent comme une lettre à la poste et dont le profane ne s'aperçoit pas.

Du fait qu'il travaille à partir de la langue parlée, le métier du traducteur de synchronisation nécessite un don de rendre de façon naturelle, synchrone et fidèle, un dialogue naturel, lui aussi. C'est pourquoi Fodor estime que "le doublage exige un haut niveau de compétence ".

Même si un synchronisateur a fait de son mieux et n'a négligé aucun des critères requis, il n'arrivera jamais au synchronisme parfait mais ce n'est pas indispensable non plus pour qu'un film synchronisé soit bon :

On ne juge pas la qualité globale d'un film synchronisé sur quelques exemples isolés de déphasage phonétique mais sur la fréquence et l'importance de ces déphasages(15).

\section{Les films de Caillé}

Quand on examine la liste des films synchronisés, adaptés ou sous-titrés par Caillé, on est d'abord frappé par leur nombre. La liste de la Société Parisienne de Sonorisation, que m'a communiquée Alain Caillé, comporte 395 films, mais ne commence qu'en 1943. Dans une interview qu'il accorda à Claude Noël, Caillé parlait de 500 films. C'est en 1936 que débuta sa carrière de synchronisateur.

À côté de grands classiques comme Jules César (1954), Électre (1963), Ben Hur (1960), Quo Vadis (1955) et Autant en emporte le vent (1950), P.-F. Caillé travailla sur toutes sortes de films. Les documentaires, comédies musicales, dessins animés, films pour la jeunesse, feuilletons télé, policiers, westerns, etc. firent partie de ses activités. Tout comme en traduction littéraire, ses talents étaient multiples. Caillé ne synchronisa pas seulement des films anglais mais aussi espagnols (Bandido Caballero - 1956), allemands (So lange Du da bist-1956), russes (Guerre et paix - 1967/68) et japonais (La porte de l'enfer, 1955). Du fait même de cette diversité, tous les films n'étaient pas d'une haute qualité, même sur le plan linguistique. Comme il a déjà été mentionné, un synchronisateur peut changer bien des choses, même le niveau du langage. Dans certains cas on le lui demande :

"Souvent on demande au synchronisateur d'éliminer l'argot de son texte, même si celui-ci en fourmille(16) ". Même si ce n'est pas le cas, la version synchronisée donne fatalement une langue d'un moindre niveau. C'est pourquoi les critiques de quelques confrères de Caillé, selon lesquels les synchronisations de ce dernier ne seraient pas écrites en "bon français " ne me semblent pas fondées. Après tout Sidney Howard n'est pas Shakespeare et Rhett Butler n'est pas Marc-Antoine!

(15) Fodor.

(16) Rowe. 


\section{Quelques exemples}

Parmi tous les films synchronisés, adaptés ou sous-titrés par P.-F. Caillé, il ne m'a pas été facile de choisir. Je n'ai malheureusement pas pu trouver son film préféré Jules César de J. Mankiewicz - avec Marlon Brando dans le rôle de Marc-Antoine - en vidéocassette, ni en anglais, ni en français, finalement je me suis décidée pour les films Autant en emporte le vent, qu'il aimait également beaucoup, et West Side Story.

\section{Autant en emporte le vent}

Comme déjà indiqué, le roman éponyme de Margaret Mitchell avait été traduit par P.-F. Caillé, qui avait reçu pour ce travail le prix Halpérine-Kaminsky. En dehors du fait que le film, comme le roman, reçurent du public français un accueil enthousiaste, le film est très intéressant pour un observateur critique de la synchronisation. Caillé utilise assez souvent sa propre traduction du roman dans les dialogues du film. D'autre part la transposition du dialecte Noir (" petitnègre ") présente de grandes difficultés. Caillé échangea avec Margaret Mitchell toute une correspondance à ce sujet.

Je voudrais citer comme premier exemple la première scène de ce film, dans laquelle on voit Scarlett de face et en gros plan. Les parties de dialogue en gras représentent les passages littéralement repris du roman ou de sa traduction.

Les positions de la caméra sont indiquées selon le code mentionné plus haut. Les parties indiquées $\S$ sont celles ou l'acteur n'est pas visible.

- Scarlett : CU/FA

- Fiddle-dee-de, war, war, war. This war-talk' s...

- Taratata, la guerre, la guerre, la guerre ces bruits...

...spoiling all the fun of everybody...

...de guerre finiront par empêcher tout le monde de... .

...this spring. I get so bored I could scream !

...s'amuser ce printemps. Ça commence à me taper sur les nerfs !

[...] On voit facilement que la version française est plus longue que l'anglaise. Néanmoins comme on parle nettement plus vite en français qu'en anglais par exemple, ce n'est qu'au passage "Ce printemps " que la version synchronisée accélère son rythme. Cette partie est " avalée " et difficile à saisir. Après " sur les nerfs " on voit, en version française, un mouvement muet des lèvres, ce qui surprend. Le responsable ne semble pas être le traducteur mais plutôt un des traitements qu'un film synchronisé doit subir. 
Un autre point qui saute aux yeux d'un spectateur, même non averti, est l'ouverture de la bouche à "la guerre ". Le mot anglais war est nettement plus ouvert que le français " guerre ", ce qui crée un déphasage phonétique très perceptible.

L'exemple suivant montre Scarlett et Ashley en gros plan et de côté :

- Scarlett : CU/SA

\section{- Oh don't tease me now. Have I your heart?}

- Oh ne vous moquez pas. Je suis certaine de l'avoir...

Puis la réponse :

- Ashley : CU/SA

\section{- You must not say such things, you will hate me for hearing them.}

- II ne faut pas dire ces choses-là. Vous m'en voudrez de vous écouter.

Dans la première phrase de Scarlett on a en français une labiodentale ([ $\mathrm{v}]$ ) et deux bilabiales ( $[\mathrm{m}],[\mathrm{p}]$ ) et rien de tel en anglais. Comme la position de la caméra CU/SA ne permet de distinguer des autres sons que les seules voyelles ouvertes, la version française ne choque pas, même un observateur attentif. Dans la deuxième phrase la question fait place en français à une affirmation mais cela ne gêne pas. Ce n'est qu'en comparant les deux versions qu'on s'aperçoit que cette divergence constitue une perte. En ce qui concerne la réponse d'Ashley on remarquera que l'original, tout comme la version française, proviennent directement du roman et de sa traduction.

La synchronisation propre à Ashley est certes convaincante mais si on la compare à l'original on voit que l'accent tonique et le timbre de la voix ne sont nullement synchrones. On ne procède cependant presque jamais à une telle comparaison et il n'y a donc pas lieu de s'en inquiéter.

Les dialogues un peu plus longs avec Mamy et Prissy, les «négresses " de Scarlett, les darkies, comme elle les appelle avec tendresse, constituent, du point de vue de la technique de la traduction, la partie sans doute la plus intéressante du film.

- Prissy :

\section{- Is de Doctah come?}

- Est-ce que l'docteur' vient?

$\S$

- Scarlett :

\section{No. He can't come.}

Non. II n'peut pas. 
- Prissy :

- Gawd, Miss Scarlett ! Mis Melly bad off.

- Oh, Ma'am Scarlett. Ma'm Melly va pas bien.

LS/SA

- Scarlett :

- He can't come. There's nobody to come.

- Nous n'pouvons compter sur personne.

MS/FA

- Prissy, you' ve got to manage without the Doctor.

- Prissy, tu vas être obligée d'te débrouiller toute seule.

$\mathrm{MS} / \mathrm{SA}$

- I'll help you.

- Je t'aiderai.

MS/FA

- Prissy :

- Oh Laudee, Miss Scarlett!

- Oh c'est affreux Ma'm Scarlett !

$\mathrm{MS} / \mathrm{SA}$

- Scarlett :

- Well, what is it?

- Mais enfin quoi, qu'y a-t-il ?

$\S$

- Prissy :

- Laudee, we's got ter hav a doctah. Ah doan know...

- C'est affreux. II faut che'cher un docteu'. Jen'sais ...

... nuthin' 'bout birthin' babies

... pas comme on fait pour les bébés.

MS/FA

- Scarlett :

- Wat do ya mean? 
- Explique-toi

CU/FA

- Prissy

- Ah doan know

- J'sais rien faire.

$\S$

- Scarlett :

- You told me you knew everything about it!

- Tu m'as pourtant dit le contraire d'vant le docteur Meade !

$\mathrm{CU} / \mathrm{FA}$

- Prissy :

- Ah doan know huccome Ah tell sech a lie. My Ma nebber lemme be'round...

- J'sais pas comment ça s'fait j'ai menti. Maman et les sages-femmes ...

...folkses whut wuz havin' them.

...m'avaient jamais voulu permettre

$\mathrm{MS} / \mathrm{FA}$

En anglais comme en français l'orthographe a été reprise du roman et de la traduction de Caillé.

En ce qui concerne la synchronisation du dialecte des esclaves on notera que Caillé concède aux Noires davantage de connaissances grammaticales que Margaret Mitchell. Leur prononciation est aussi davantage compréhensible. Dans cette scène l'effet optique est impeccable mais il faut remarquer qu'il n'y a que deux positions CU/FA de la caméra et que le décor (une cage d'escalier) est plutôt sombre, ce qui réduit la possibilité de déceler des déphasages phonétiques.

Le synchronisme interne est ici parfait ainsi que le synchronisme textuel, si on oublie que laversion française est pleine d'additions : "le Docteur Meade " et "les sages-femmes ".

Cet exemple de dialogue permet d'illustrer un des " trucs " habituels des synchronisateurs.

Les deux textes soulignés dans la version française sont dits en même temps, alors que ce n'est pas le cas en anglais.

C'est possible parce que Scarlett n'est pas visible à : "Mais enfin quoi... ". On peut ainsi remplacer l'exclamation anglaise par une exclamation française presque deux fois plus longue. 
Le dernier exemple tiré de ce film est intéressant du point de vue phonétique :

- Mamy :

- Mornin', Miss Melly...

- Bonsoir, Ma'm Melly ...

CU/FA

Sur le plan textuel le déphasage est évident mais on n'a pas le temps de déceler le déphasage interne car le texte est trop court. Du point de vue phonétique on comprend le déphasage textuel : il était impossible de traduire mornin par "bonjour ", sans accepter un déphasage gênant, même pour le profane. Comme le décor où se situe le dialogue est sombre, cette traduction ne semble pas absurde. Par ailleurs dans le roman, contrairement au film, le dialogue entre Mammy et Melany a lieu effectivement le soir.

\section{West Side Story}

J'ai choisi ce film pour trois raisons :

1. - nous rencontrons ici deux types de langues (l'américain et le portoricain), ce qui du point de vue linguistique et " interne " est intéressant ;

2. - le texte parlé par les Américains est presque exclusivement de l'argot ;

3. - j'ai trouvé dans ce film un dialogue entièrement constitué de mots isolés, ce qui présente une difficulté particulière. En version française les chansons ne furent pas traduites :

- Schrank :

- How many times have I told you punks ...

- Combien de fois est-ce que je vous ai dit...

LS/FA

- Riff :

- Why if it aint Lieutenant Schrank!

- Ah mais c'est le lieutenant Schrank !

LS/SA

- Jets :

- Top a da day, lieutenant Schrank!

- On v'souhaite une bonne journée, Lieutenant Schrank !

LS/SA 
- Bernardo :

- And Officer Krupke!

- Et le sergent Krupke!

$\mathrm{LS} / \mathrm{SA}$

- Krupke :

- l' Il give ya a top a the head(17).

- C'est moi qui vais vous la souhaiter!

LS/FA

- Schrank :

- Get ya ... "friends"... outa here, Bernardo...

- Toi et tes potes derrière vous, Bernardo...

CU/FA

... and stay out.

...n'remettez plus les pieds ici.

$\S$

Please.

S'il vous plaît.

CU/FA

Le texte original provient du livret (script) que la Cinémathèque Française a bien voulu mettre à ma disposition. Par endroits le texte a été modifié au tournage. Les citations reproduites ici suivent le dialogue réel du film.

Dans ce dialogue aussi on remarque que le style de la version française est d'un niveau supérieur à celui de l'original. Le ton est cependant préservé. Pour traduire de tels films le synchronisateur doit maîtriser tous les registres de la langue-source et de la langue-cible. Dans la première partie de cet exemple le synchronisme est parfait. La version française est constamment " dense ", ce qui favorise le synchronisme interne. La dernière phrase du Lieutenant Schrank présente quelques anomalies :

(17) Top da a day, Officer Krupke! - l'll give ya a top a the head.

Le jeu de mot est intraduisible ... et n'a pas été traduit ! - N. du T. 
"Toi et tes potes derrière vous... ". II emploie le " toi " et le " vous " dans la même phrase, ce qui crée un déphasage textuel qui choque même si on ne compare pas à l'original. Ce choix surprend d'autant plus qu'il n'apporte pas de synchronisme phonétique, cependant que le synchronisme interne n'est pas pris en considération.

La longueur bien plus grande de la phrase française s'explique par le fait que Schrank n'est plus visible à l'écran lorsqu' il termine sa phrase. Le Please isolé et, de plus, en gros plan est pour le synchronisateur "incontournable ". S'il ne veut pas risquer un décalage textuel et interne aisément décelable, il lui faut sacrifier l'aspect phonétique-optique.

J'ai choisi l'extrait suivant uniquement parce qu'il me semble être un modèle de bonne synchronisation. Le dialogue est naturel, le synchronisme interne rendu par la vitesse de l'élocution et par l'accent et le synchronisme textuel parfaitement respecté. Sur le plan phonétique je n'ai découvert aucun déphasage et il est vrai que les positions de la caméra sont favorables.

Comme dans l'exemple précédent, le texte est un peu dense, ce qui ne fait que favoriser le synchronisme interne :

- Maria :

- You must make the neck lower!

- Rabaisse un peu le décolleté !

CU/FA

- Anita :

- And you must stop heckling me. We are working on our time now and not...

- Est-ce que tu as fini de me casser les pieds, on travaille à notre ...

- ..the old lady's.

- ...compte, pas à celui de notre patronne.

MS/FA

- Maria :

- One inch,

- Un centimètre

$\S$

- How.much can one little inch do ?

- Ça ferait une grosse différence, tu crois ?

MS/FA 
- Anita :

- Too much.

- Très grosse.

$\mathrm{MS} / \mathrm{BA}$

Le dernier exemple illustre la difficulté de bien synchroniser des mots lancés isolément, parfois par des personnages en gros plan:

- Bernardo :

- Your challenge.

- À toi de choisir.

$\mathrm{MS} / \mathrm{SA}$

- Riff :

- Afraid to call?

- Ça t'fait peur?

- Bernardo :

- Rocks

- Tuyaux

MS/FA

- Riff :

- Belts.

- Ceintures.

MS/FA

- Bernardo :

- Pipes

- Matraques.

- Riff :

- Cans.

- Caisses.

MS/FA

- Bernardo :

- Bricks.

- Briques. 
MS/FA

- Riff :

- Bats.

- Pierres.

$\mathrm{MS} / \mathrm{FA}$

- Bernardo :

- Clubs

- Cannes.

MS/FA

Quand Caillé, pour des raisons optiques, renonce au synchronisme textuel, cela semble, trois fois sur quatre, justifié. Rocks et "tuyaux " sont proches phonétiquement. De même cans " caisses " et bats - "pierres ". Dans le quatrième cas il n'y a plus de synchronisme interne ni phonétique : "Clubs " comporte un son bilabial qu'on peut nettement distinguer de tous les autres, surtout avec la caméra en position MS/FA. Le mot "cannes " est absolument déphasé, car dépourvu d'un pareil son.

Pour finir je voudrais préciser que j'ai consacré la majeure partie du temps que j'ai passé devant l'écran à chercher des passages où il pouvait y avoir un déphasage. Exception faite du mauvais début d'Autant en emporte le vent, en tant que "spectatrice moyenne ", je n'aurais été gênée par aucun passage. Si toutefois on voit la version originale aussitôt après la version synchronisée et si on les compare, si par ailleurs on dispose des documents écrits, alors évidemment les divers " trucs " et anomalies sautent aux yeux.

Pour ce qui est de l'impression générale qu'on éprouve en voyant les films doublés par P.-F. Caillé, on peut, selon moi, considérer son nom comme une garantie de bonne synchronisation.

\section{Note bibliographique en postface à la publication de 2014}

\section{Pierre-François Caillé (1907-1979)}

Brillant traducteur littéraire, il a traduit de nombreux romans (de Margaret Mitchell comme chacun sait mais aussi de Mark Twain ou Louis Bromfield, entre autres) et de nombreux dialogues de film comme en atteste l'article ci-dessus.

Spécialiste des questions de droit d'auteur, expert près de l'Unesco, il est l'un des fondateurs de la Société française des traducteurs (SFT), en 1947, et de la Fédération internationale des traducteurs (FIT), en 1953, ainsi que de la revue Babel. Il a également écrit des articles sur la traduction, et en particulier : "Cinéma et traduction : le traducteur devant l'écran - Le dou- 
blage - Le sous-titrage ", Babel, vol. 6, n 3, 1960, p. 103-109 et "La traduction au cinéma ", in R. Italiaander (dir.), Übersetzen. Vorträge und Beiträge vom internationalen Kongress literarischer Übersetzer in Hamburg, Hambourg, Francfort, Bonn, Athenäum, 1965, p. 116-122.

En 1980, la SFT entreprend de créer un prix à la mémoire de celui qui a été son président pendant 23 ans : ce sera le Prix Pierre-François Caillé de la traduction (voir à ce propos l'article de Florence Herbulot, elle-même présidente du jury pendant près de trente ans, dans le numéro 221 de Traduire, disponible en ligne à l'adresse http://traduire.revues.org/358). Le Prix PierreFrançois Caillé de la Traduction est décerné chaque année en décembre. Depuis décembre 2013, le jury est présidé par Débora Farji-Haguet.

Edmond Cary (1912-1966), cité à plusieurs reprises dans l'article, interprète et traducteur, a participé, avec Pierre-François Caillé, à la création de la SFT et de la FIT ; traductologue, il a également contribué - toujours avec Pierre-François Caillé - à la fondation de la revue internationale de traduction Babel (publiant dans le premier numéro un article sur Étienne Dolet). II est notamment l'auteur des Grands traducteurs français(18). Comment faut-il traduire ?(19) est un ouvrage posthume qui rassemble huit cours radiophoniques donnés en 1958 ; citons encore sur le thème de la traduction au cinéma : "La traduction cinématographique ", in La traduction dans le monde moderne, E. Cary (dir.), Genève, Librairie de l'Université, Georg S.A., 1956, p. 105-113 ; "La traduction totale ", Babel, vol. 6, n 3, 1960, p. 110-115 et "Comment s'effectue le doublage cinématographique ", in Comment faut-il traduire ?, Presses universitaires du Septentrion, Lille, 1985, p. 65-71.

Christian Delmas, auteur de "Les traductions synchrones ", in La traduction, une profession, Paul A. Horguelin (dir.), Actes du VIIIle Congrès mondial de la FIT, 1978.

István Fodor, auteur de "Linguistic and psychological problems of film synchronization ", in Acta Linguistica Academiae Scientarium Hungaricae, 19 (2 \& 3-4), 1969, Budapest, p. 69-106 et 379-394 et Film Dubbing - Phonetic, Semiotic, Esthetic and Psychological Aspects(20); il fut l'un des premiers à décrire les différents types de synchronisation et à étudier une " phonétique visuelle " visant à faire correspondre les mouvements de la bouche de l'acteur sur l'écran et les phonèmes nécessaires à la traduction, de manière à éviter les déphasages.

Thomas L. Rowe, auteur de "The English dubbing text ", Babel 6, p. 116-120, 1960.

Hans Vöge, auteur de "The translation of films: subtitling versus dubbing " in Babel23, p. 120125, 1977.

(18) Genève, Georg, 1963.

(19) Presses universitaires du Septentrion, Lille, 1985.

(20) Hamburg: Helmut Buske Verlag, 1962 (1re édition ; 2e édition 1969 ; 3e édition 1976). 